\title{
Review \\ Biomarkers of endothelial activation and dysfunction in cardiovascular diseases
}

\author{
Jun Zhang ${ }^{1, *}$ \\ ${ }^{1}$ Baylor Heart and Vascular Institute, Dallas, TX 75226, USA \\ *Correspondence: Zhangj37@gmail.com (Jun Zhang) \\ Academic Editors: Carmela Rita Balistreri and Davide Bolignano \\ Submitted: 25 October 2021 Revised: 8 November 2021 Accepted: 17 November 2021 Published: 22 February 2022
}

\begin{abstract}
Endothelial activation and dysfunction is an important contributor to atherosclerosis, cardiovascular diseases and cardiorenal syndrome. Endothelial dysfunction is also linked with metabolic syndrome and type II diabetes. The search for specific and sensitive biomarkers of endothelial activation and dysfunction may have important clinical implications. This review pinpoints the differences in biomarkers between endothelial activation and endothelial dysfunction in cardiovascular diseases, and then briefly describes the most relevant biomarkers of endothelial activation. Biomarkers of endothelial activation include endothelial adhesion molecules, cytokines, C-reactive protein, $\mathrm{CD} 62 \mathrm{E}^{+} / \mathrm{E}$-selectin activated endothelial microparticles, oxidation of low density lipoproteins, asymmetric dimethylarginine and endocan. This review also presents an update on the novel biomarkers of endothelial dysfunction, such as matrix metalloproteinases (e.g., MMP-7, MMP-9), ANGPTL2, endogdlin, annexin $\mathrm{V}^{+}$endothelial apoptotic microparticles, and serum homocysteine. Finally, this review emphasizes the limitations of biomarkers of endothelial activation and dysfunction in clinical setting.
\end{abstract}

Keywords: biomarkers; cardiovascular diseases; endothelial activation; endothelial dysfunction

\section{Introduction}

Endothelial activation and dysfunction is an important contributor to atherosclerosis, cardiovascular diseases (CVD) (e.g., coronary artery disease, carotid artery disease, peripheral artery disease, and ischemic stroke) [1, 2] and metabolic syndrome [3]. Recently it is proposed that endothelial and insulin signalling pathways crosstalk each other. The relationship between endothelial function and insulin metabolism is very important to hypertension, obesity, and diabetes. Insulin resistance, a hallmark of metabolic syndrome, impairs vascular response and increases cardiovascular risk. Insulin resistance and endothelial dysfunction in these pathological disorders result in impairment in the nitric oxide - dependent vasodilatation, cellular glucose uptake, enhancement in oxidative stress, and inflammation. Finally, all these lesions lead to atherosclerosis [4]. Moreover, it is widely known that insulin resistance is also a hallmark of prediabetes, which can eventually lead to type II diabetes. Therefore, insulin resistance, like other risk factors for atherosclerosis (e.g., hypertension and hypercholesterolemia), promotes endothelial dysfunction, which then leads to development of metabolic syndrome [4]. More recently it is suggested that endothelial dysfunction is linked with metabolic syndrome and type II diabetes [5]. The search for specific and sensitive biomarkers of endothelial activation and dysfunction may have broader clinical significance, because CVD remains the leading cause of morbidity and mortality in the United States. Recently there has been considerable interest in the search for novel, potential biomarkers of endothelial acti- vation and dysfunction in CVD. At present it has been the emergence of novel candidate biomarkers as potential diagnostic or predictive risk factors for CVD. These efforts could gain insight into pathogenesis for CVD, and the insight may lead to new approaches in treatment of CVD as well. In this review, we give an update of the current advances on emerging candidate biomarkers of endothelial activation and dysfunction.

\section{The differences in biomarkers between endothelial activation and endothelial dysfunction in CVD}

Although the term 'endothelial dysfunction' is widely used in CVD, it has been known for long time that endothelial activation, as exemplified by the type I (endothelial stimulation) and type II endothelial activation, is the initial event leading to endothelial dysfunction [1,6] (Fig. 1). The issue of endothelial proinflammatory activation and endothelial dysfunction in atherogenesis was more fully discussed in a review by Gimbrone and Garcia-Cardefia [1]. Defining the sequence of initial proinflammatory activation and subsequent dysfunction of the endothelium may provide necessary insight to understand the cellular and molecular mechanisms of CVD. Moreover, the sequence of events can make several linkages between endothelial activation and dysfunction in the development of atherosclerosis: (1) a link between acute inflammatory responses (preceding alterations in the development of atherosclerotic change) and chronic inflammatory process (within the endothelial lining in atherosclerosis susceptible regions); (2) a link be- 


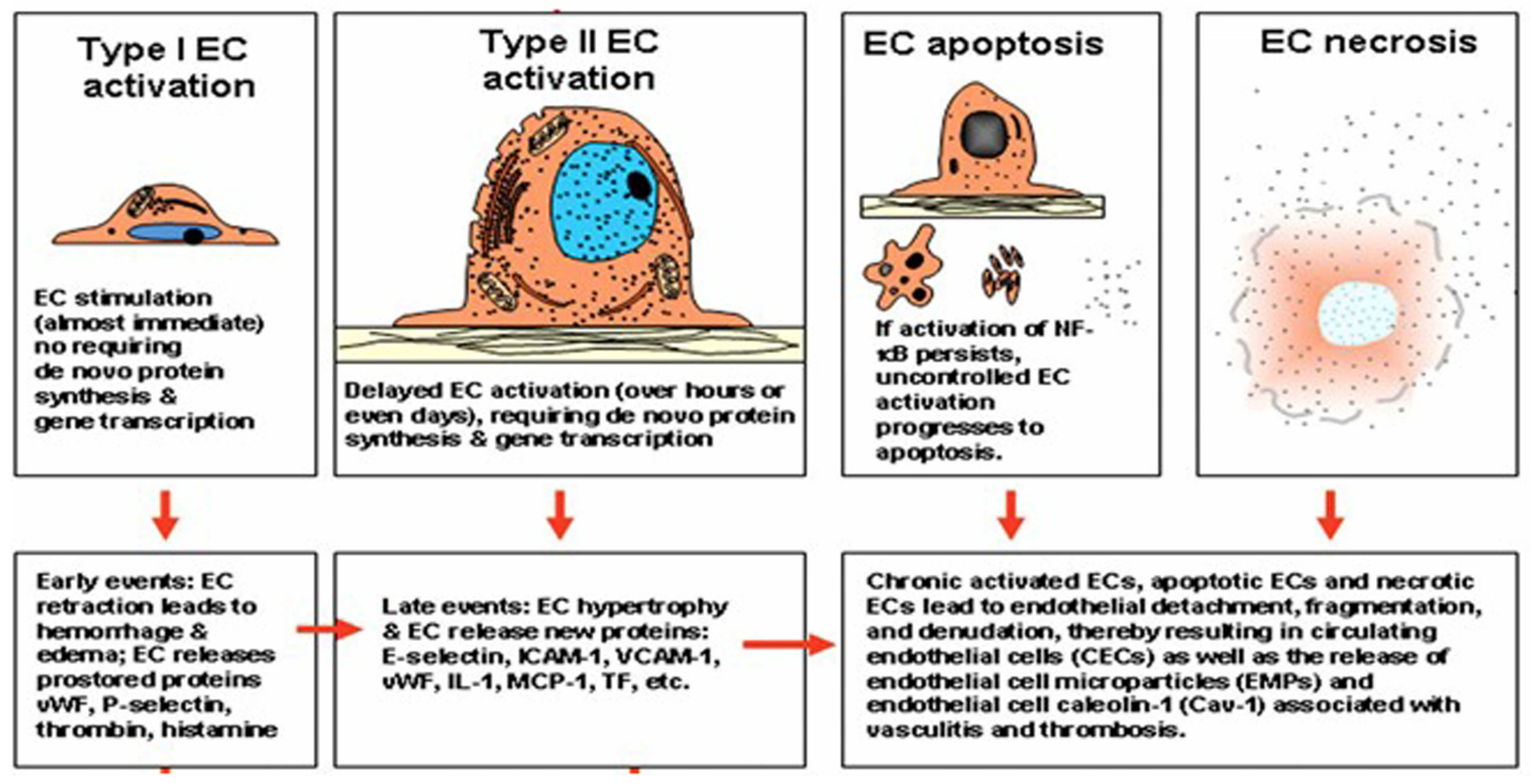

Fig. 1. Schematic representation of the relationship of endothelial cell (EC) activation and endothelial dysfunction. Endothelial cell activation consists of type I and type II EC activation, whereas endothelial dysfunction consists of EC apoptosis and EC necrosis (a hallmark of endothelial injury). The EC activation process, if uncontrolled, can progress to endothelial dysfunction. Type I EC activation (immediate event) and Type II EC activation (over hours or days) are reversible when EC activators are withdrawn, whereas chronic endothelial activation (over months) leads to EC injury with EC detachment from the underlying basement membrane and denudation of the vessel wall, resulting in circulating endothelial cells and the release of EC microparticles (EMPs) and EC caveolin-1 (Cav-1) from the plasmalemmal membranes. TNF, tumor necrosis factor; IL-1, interleukin-1; IFN, interferon; LPS, lipopolysaccharide. Adopted from Zhang J, Defelice AF, Hanig JP, Colatsky T. Biomarkers of endothelial cell activation serve as potential surrogate markers for drug induced vascular injury. Toxicologic Pathology. 2010; 38: 856-871.

tween the microvasculature (e.g., post-capillary venules, small vessels, small arteries) and the macrovasculaure (e.g., large arteries); and (3) a link between localized atherosclerosis and systemic extensive atherosclerosis.

The linkage of activation biomarkers with dysfunction biomarkers can be exemplified in experiments of in vivo (APoE/LDLR double knockout mice) and in vitro (human aortic endothelial cells), in which cholesterol [hyperchoclesterolemia and 7-ketocholesterol (7K)] induced not only activation biomarkers (e.g., E-selectin, P-selectin, ICAM-1, VCAM-1), but also induced a novel biomarker of endothelial dysfunction, endoglin [7]. These findings suggest that activation biomarkers positively correlated with dysfunction biomarker in the face of potent systemic drivers of cardiovascular risk (e.g., hypercholesterolemia) [7]. Indeed, Lundman et al. [8] previous demonstrated higher levels of soluble E-selectin and soluble ICAM-1 (hallmarkers of endothelial activation) in the hypertriglyceridemic group than in the control group. Moreover, endothelial microparticles are vesicles released from activated cells or apoptotic cells. These microparticles are composed of the plasma membrane vesicles or plasma membrane blebbing detached from vascular endothelium. Upon detached, these vesicles enter the circulation and serve as a biomarker of endothelial activation or biomarker of endothelial dysfunction. Endothelial microparticles as biomarker of endothelial activation can be exemplified by findings of microparticles captured with anti-CD62 $\mathrm{E}^{+} / \mathrm{E}-$ selectin, anti-CD54 ${ }^{+} / \mathrm{ICAM}-1$, and anti-D106 ${ }^{+}$VCAM-1, whereas endothelial microparticles as biomarker of endothelial dysfunction (e.g., endothelial apoptosis and damage) is evidenced by findings of macroparticles captured with anti-annexin $\mathrm{V}$ to phosphatidylserine [9-12].

\section{Biomarkers of endothelial activation in cardiovascular diseases}

Table 1 represents circulating soluble and cell component biomarkers of endothelial activation in cardiovascular diseases. Biomarkers of endothelial activation include a heterogeneous group of candidate molecules. Although endothelial cells of various organs possess many common functional and morphological features, endothelial cells clearly exhibit significant heterogeneity of constitutive and inducible molecules across organs and, in the same organ, across vessels (post-capillaries, venules, arterioles, arteries, small vessels, and large vessels). Therefore, 
Table 1. Representative circulating soluble and cell component biomarkers of endothelial activation in cardiovascular diseases.

\begin{tabular}{|c|c|c|c|c|}
\hline Biomarkers & Alternative names & $\begin{array}{l}\text { Family/Super } \\
\text { family }\end{array}$ & Major cellular sources & Potential use \\
\hline E-selectin & $\begin{array}{c}\text { CD62E, } \\
\text { endothelial-leukocyte } \\
\text { adhesion molecule-1 } \\
\text { (ELAM-1) }\end{array}$ & Selectin family & $\begin{array}{c}\text { Exclusively from activated } \\
\text { endothelium, as a conclusive evidence } \\
\text { of endothelial activation }\end{array}$ & $\begin{array}{l}\text { A sensitive and specific marker } \\
\text { for endothelial activation }\end{array}$ \\
\hline P-selectin & $\begin{array}{l}\text { CD62P, GMP-140, } \\
\text { PADGEM }\end{array}$ & Selectin family & $\begin{array}{l}\text { Weibel-Palade dies of activated } \\
\text { endothelium }\end{array}$ & $\begin{array}{l}\text { A sensitive marker for } \\
\text { endothelial activation }\end{array}$ \\
\hline ICAM-1 & $\begin{array}{l}\text { CD54, intercellular } \\
\text { adhesion molecule-1 }\end{array}$ & $\begin{array}{l}\text { IgG gene } \\
\text { superfamily }\end{array}$ & Activated endothelium & $\begin{array}{l}\text { A reliable, sensitive marker } \\
\text { for endothelial activation }\end{array}$ \\
\hline VCAM-1 & $\begin{array}{l}\text { CD106, vascular cell } \\
\text { adhesion molecule- } 1\end{array}$ & $\begin{array}{l}\text { IgG gene } \\
\text { superfamily }\end{array}$ & Activated endothelium & $\begin{array}{l}\text { A reliable , sensitive marker } \\
\text { for endothelial activation } \\
\text { Activated endothelium }\end{array}$ \\
\hline IL-6 & Interleukin-6 & Cytokine family & Activated endothelium & $\begin{array}{l}\text { A reliable, sensitive marker for } \\
\text { endothelial activation activated } \\
\text { endothelium, nonspecific }\end{array}$ \\
\hline CRP & C-reactive protein & $\begin{array}{l}\text { Acute phase } \\
\text { protein }\end{array}$ & Activated endothelium & $\begin{array}{l}\text { A reliable, sensitive marker for } \\
\text { endothelial activation activated } \\
\text { endothelium, nonspecific }\end{array}$ \\
\hline LOX-1 & $\begin{array}{l}\text { Lectin-like oxidized LDL } \\
\text { receptor-1, }\end{array}$ & $\begin{array}{c}\text { A receptor for } \\
\text { oxidized (ox) LDL }\end{array}$ & Activated endothelium & $\begin{array}{l}\text { A promising novel marker for } \\
\text { endothelial activation, } \\
\text { nonspecific }\end{array}$ \\
\hline CD40L & $\begin{array}{l}\text { CD ligand, CD154, } \\
\text { TNF-related activation } \\
\text { protein }\end{array}$ & TNF superfamily & Activated endothelium & $\begin{array}{l}\text { A promising novel marker for } \\
\text { endothelial activation, } \\
\text { nonspecific }\end{array}$ \\
\hline ADMA & $\begin{array}{l}\text { Asymmetric dimethyl } \\
\text { arginine }\end{array}$ & $\begin{array}{l}\text { An endogenous } \\
\text { NOS family }\end{array}$ & Activated endothelium & $\begin{array}{c}\text { A promising novel marker for } \\
\text { endothelial activation, } \\
\text { nonspecific }\end{array}$ \\
\hline Endocan & $\begin{array}{c}\text { Endothelial cell-specific } \\
\text { molecule }-1\end{array}$ & The proteoglycan & Activated endothelium & $\begin{array}{l}\text { A promising novel marker for } \\
\text { endothelial activation }\end{array}$ \\
\hline EMPs & $\begin{array}{l}\mathrm{CD} 62 \mathrm{E}^{+} \text {endothelial } \\
\text { microparticles }\end{array}$ & & Activated endothelium & $\begin{array}{l}\text { A promising novel marker for } \\
\text { endothelial activation }\end{array}$ \\
\hline
\end{tabular}

Data presented here from Zhang J, Defelice AF, Hanig JP, Colatsky T. Biomarkers of endothelial cell activation serve as potential surrogate markers for drug induced vascular injury. Toxicologic Pathology. 2010; 38: 856-871.

a long list of potential biomarkers of endothelial activation is available, as documented by review in CVD $[1,6,9]$, in kidney disease [10], in peripheral vascular disease [11] and in vascular injury in animals [12]. The data presented here briefly highlight some circulating soluble and cell component biomarkers of endothelial activation in CVD.

Biomarkers of endothelial activation may provide information about a risk in the development of cardiovascular disease and new targets for treatment [13]. In the context of atherogenesis, the search for biomarkers of endothelial activation is of great significance in that endothelial activation precedes and may stimulate the development of atherosclerotic lesions [9]. In atherosclerotic cardiovascular disease, the proinflammatory endothelial activation results in the release of endothelial adhesion molecules (e.g.,
E-selectin, P-selectin, ICAM-1, and VCAM-1) [1]. These adhesion molecule expression is induced by proinflammatory cytokines such as IL- $1 \beta$ and tumor necrosis factor- $\alpha$ $(\mathrm{TNF}-\alpha)$. Other inducers of adhesion molecules may be through the acute-phase protein (CRP) that is produced by the liver in response to interleukin-6 (IL-6) [13].

Hwang et al. [14] studied 272 patients with carotid artery atherosclerosis, 204 patients with incident coronary heart disease, and 316 control subjects. They found that higher levels of E-selectin and ICAM-1 were observed in the patients with coronary heart disease and those with carotid artery atherosclerosis. In addition, levels of Eselectin were significantly correlated with high-density lipoprotein- cholesterol in the control group. These findings indicate that E-selectin may be involved in the early steps 
of atherosclerosis by mediating initial rolling of leukocytes along the endothelium, and levels of circulating ICAM-1 are more closely related to the activity of atherosclerosis by its roles in the firm attachment and transendothelial migration of leukocytes. Therefore, Hwang et al. [14] suggest that soluble E-selectin and ICAM-1 may serve as biomarkers of endothelial activation for carotid atherosclerosis and subclinical coronary heart disease. In a population-based cohort study, Tzoulaki et al. [15] compared the association between biomarkers of endothelial activation (e.g., soluble adhesion molecules such as sICAM-1, sVCAM-1, and sE-selectin) and progression of peripheral atherosclerosis. They found that sICAM-1, IL-6, and CRP were significant predictors of lower-extremity atherosclerotic progression. They suggest that ICAM-1, IL-6, and CRP are molecular markers associated with atherosclerosis and its progression [15]. It is worth noting that although increased circulating levels of ICAM-1 and VCAM-1 were considered a consequence of endothelial activation, E-selectin is expressed solely on the activated endothelium, therefore, its presence in the circulating blood is taken as conclusive evidence of endothelial activation $[11,12]$.

The proinflammatory activation of endothelial cells would be an important source of IL-6 generation. Highsensitivity C-reactive protein (hsCRP) by endothelialderived IL-6 is now considered as a robust, clinical useful biomarker in the assessment of risk factor for atherosclerotic cardiovascular disease [1]. Furthermore, IL-6 and Creactive protein is correlated with circulating microparticles in patients with coronary heart disease [15]. Cui et al. [16] analyzed circulating endothelial microparticles in 40 patients with myocardial infarction, 30 unstable angina, 20 stable angina and 20 healthy individuals. They demonstrated that endothelial microparticles were significantly elevated in myocardial infarction and unstable angina, as well as the elevated levels of endothelial microparticles were correlated with IL-6 or CRP level. Their results indicate that endothelial microparticles may participate in the development of thrombosis and enhance the vascular inflammation [16].

Elevated circulating levels of endothelial microparticles were found in patients with acute coronary syndrome, diabetes mellitus and severe hypertension, and thus endothelial microparticles could be used as a surrogate marker in patients with CVD [9]. There is growing consensus that endothelial microvesicles may serve as a novel biomarker foe CVD [1,10,17-19]. Endothelial microvesicles, which are shredded from the activated endothelium through cell detachment and loss, are potentially used as endothelial activation biomarker for atherosclerosis [1]. Rabelink et al. [10] suggest that circulating levels of endothelial microparticles could serve as a biomarker of sustained endothelial activation. In concert of this suggestion, Berezin also pointed out that circulating endothelialderived microparticle is a novel biomarker of cardiovas- cular risk [17]. The surface of endothelial-derived microparticles expresses membrane proteins such as tissue factor, E-selectin, and P-selectin (hallmarkers of endothelial activation) [17]. In this context, circulating endothelialderived microparticles could serve as a marker of endothelial activation. On the other hand, the surface of these microparticles can also express annexin V to phosphatidylserine (a hallmark of apoptotic endothelial cells), and thus the microparticles could also be a marker of endothelial dysfunction. From pathological perspective $[1,11,12]$, activated endothelial - derived microparticles is followed by apoptotic endothelial-derived microparticles, indicating progress of endothelial cells from early mild perturbation to late severe injury. Elevated $\mathrm{CD}^{2} 2 \mathrm{E}^{+}$- Circulating endothelial microparticles captured with elevated levels of $\mathrm{CD} 2 \mathrm{E}^{+}$was presented in patients with ischemic stroke and transient ischemic attack [16]. Hu et al. [18] demonstrated that the mean percentage of $\mathrm{CD}_{2} \mathrm{E}^{+}$microparticles was higher in the coronary artery disease patient group than in the healthy subject group. They analyzed ROC curve for $\mathrm{CD}^{2} 2 \mathrm{E}^{+}$microparticles and the cut off value of $1.35 \%$ had a sensitivity of $76.9 \%$ and specificity of $88.9 \%$. These findings indicate that endothelial activation participates in the pathogenesis of coronary artery disease by using E-selectin molecule [18]. Recently Landers-Ramos et al. [19] revealed that the number of $\mathrm{CD}^{2} 2 \mathrm{E}^{+}$endothelial microparticles was higher in the coronary artery disease ( $265.6 \pm 96.1$ particles $/ \mu \mathrm{L})$ compared with the healthy subjects $(167.6 \pm 32.1$ particles $/ \mu \mathrm{L})$, but this did not reach statistical significance. The reasons for this contradiction may be due to smaller sample sizes used in this study and a significant difference in the total number of medications taken between patients with coronary artery disease $(9.4 \pm 1.3)$ compared to healthy controls ( $4 \pm 1.1)$ [19]. Moreover, endothelial microparticles were also found in peripheral vascular diseases (e.g., microscopic polyangiitis, polyarteritis nodosa, Takayasu arteritis etc.) [12,13]. Taken together, these data indicate that endothelial microparticles could serve as a biomarker of endothelial activation. The elevated or the decreased levels of endothelial microparticles could be used to monitor the disease activity and the therapy effect.

Many other molecules released from activated endothelium have been proposed as novel biomarkers of endothelial activation for atherosclerosis and other cardiometabolic diseases (e.g., hypertension, diabetes mellitus, coronary artery disease, etc.) [9,10]. Among these proposed biomarkers, Oxidation of low density lipoproteins (Ox-LDL), asymmetric dimethylarginine (ADMA) and endocan (endothelial cell specific molecule-1) are of specific interest.

Oxidized low-density lipoprotein (oxLDL) causes endothelial activation [13]. Ox-LDL, an oxidative stress biomarker has been identified as a pro-atherogenic risk factor for coronary artery disease [9]. Lectin-like oxidized- 
low-density lipoprotein (LDL) receptor-1 (LOX-1) is the major receptor for oxidized LDL (oxLDL), which is expressed on endothelial cells. Oxidized LDL activates endothelial cells by binding to LOX-1. In addition, oxLDL, through LOX-1, triggers the CD40/CD40L signaling pathway, and thus reinforces local inflammation [11].

ADMA is an endogenous nitric oxide synthase (NOS) inhibitor, which is associated with vascular endothelial activation. In children and young adults with hypertension, increased plasma ADMA is accompanied by increased VCAM-1. The endothelium-derived NO, when inhibited, can induce vascular EC activation. In this regard, the raised plasma endogenous NO inhibitor ADMA indicates that endothelial activation is triggered by eNOS inhibition. In this regard, ADMA may serve as a specific biomarker of endothelial activation, and it could be indicative of a risk factor for CVD (e.g., hyperlipidemia, hypertension, coronary artery disease, unstable angina, stroke, and end-stage renal diseases and diabetes) [9]. On the other hand, reduced plasma levels of ADMA after percutaneous coronary intervention could be indicative of a reduced risk of recurrent cardiovascular events [9].

Endocan (endothelial cell-specific molecule-1, the proteoglycan) is an emerging novel biomarker of endothelial activation in CVD $[9,20]$. Tadzic et al. [20] studied the effect of blood pressure reduction on the profiles of endocan and soluble cell adhesion molecules in hypertensive subjects treated with Ca-channel blocker, amlodipine. The important findings include (1) serum levels of sICAM-1 and sVCAM-1 were significantly decreased after the treatment; (2) the serum endocan concentration was also decreased after the amlodipine therapy and this decrease levels of endocan tended to decrease with blood pressure reduction; and (3) the decreased levels of endocan significantly positively correlated with lower levels of ICAM-1. These findings suggest that in essential hypertension, endocan could be secreted by activated endothelium, and is able to compete with ICAM-1 for LFA-1. In contrast, de-activation of endothelial cells is associated with reduction in blood pressure. As a result, the decreased levels of endocan can indicate the decrease of the risk for development of atherosclerosis [20].

\section{Novel biomarkers of endothelial dysfunction in cardiovascular diseases}

Novel soluble and cell component biomarkers of endothelia dysfunction in cardiovascular diseases are presented in Table 2 (Ref. [21-29]). Recently, Boumiza et al. [21] studied the relationship between matrix metalloproteinases (MMPs) or tissue inhibitor of metalloproteinases (TIMPs) levels and endothelial dysfunction in 185 obese, 67 overweight, and 227 non-obese subjects. They emphasized that MMP-9 levels or MMP-9/TIMP-1 ratio could be predictive for endothelial dysfunction [21]. They proposed using MMPs and TIMPs as clinical biomarkers in obesity-related cardiovascular diseases such as metabolic syndrome and hypertension. It has generally been assumed that there is association between MMPs, TIMPs and obesity as well as obesity-related disease including metabolic syndrome. Endothelial dysfunction is one of the early determinants for obesity-related diseases including hypertension. MMPs are now thought to involve in physiological and pathological complications of obesity or metabolic syndrome. MMP-9 belongs to one member (e.g., gelatinase) of MMP family. Therefore, alterations in MMPs/TIMPs mediated-proteolysis may contribute too many pathological states (metabolic syndrome and hypertension) [21]. Their findings will hopefully provide new aspects for the use of MMPs and TIMPs as clinical biomarkers in obesity-related cardiovascular diseases such as metabolic syndrome and hypertension. In the study by Boumiza et al. [21] parameters measured included flow-mediated dilation, microvascular cutaneous reactivity hyperemia (using Laser Doppler) and acetylcholine chloride -induced microvascular vasodilatation (Laser Doppler iontophoresis) and cutaneous vascular conductance. The methodology is usually used to test endothelial dysfunction. The linkage of data of endothelial dysfunction obtained by the above methods with data of MMP and TIMP provides scientifically logical evidence of MMP and TIMP as biomarker of endothelial dysfunction are. Study by Olejarz et al. [22] is consistent with the notion of MMP-7 and MMP-9 as biomarkers of endothelial dysfunction for atherosclerotic plaque instability. It is now well recognized that MMPs can be secreted by endothelial cells. MMP-9 (Matrilysin-1) participates in endothelial dysfunction by several pathways, such as increase of inflammatory cell migration and invasion into the arterial wall, participation in oxLDL effect, and endothelial apoptosis, whereas MMP-7 (Gelatinase-B) plays a key role in development of plaque, neovascularization, calcification, as well as plaque activation and destabilization [22]. Increased activity of MMP-7 and MMP-9 was observed in unstable plaques, and the highest tissue expression of MMP-9 was found in plaques of lipid type compared with plaques of necrotic and inflammatory-erosive types [22].

ANGPT L2 is a proinflammatory mediator, which promotes vascular inflammation and atherosclerosis [23, 24]. Horio et al. [23] reported that ANGPTL2 levels in aorta tissue from the apolipoprotein E (ApoE-/-) knockout mouse were positively correlated with atherosclerosis severity. ANGPTL2 is abundantly expressed in endothelial cells and macrophages infiltrating atheromatous plaques. ANGPTL2 promotes vascular inflammation likely via a signaling pathway of nuclear factor (NF)- $\kappa \mathrm{B}$ in endothelial cells and increased monocyte/macrophage chemotaxis. Horio et al. [23] suggest that endothelial cell - derived ANGPTL2 accelerates vascular inflammation, leading to endothelial dysfunction and atherosclerosis development. Hata et al. [24] analyzed serum ANGPTL2 concentrations in 3005 community-dwelling Japanese aged $\geq 40$ years 
Table 2. Novel soluble and cell component biomarkers of endothelia dysfunction in cardiovascular diseases.

\begin{tabular}{|c|c|c|}
\hline Biomarkers & Alternative names & Potential use \\
\hline MMPs/TIMPs & $\begin{array}{l}\text { Matrix metalloproteinases/tissue inhibitor of } \\
\text { metalloproteinases }\end{array}$ & $\begin{array}{l}\text { MMP-9 levels or MMP-9/TIMP-1 ratio could be } \\
\text { predictive for endothelial dysfunction; using MMPs } \\
\text { and TIMPs as clinical biomarkers in obesity-related } \\
\text { cardiovascular diseases such as metabolic syndrome } \\
\text { and hypertension [21]; MMP-7 and MMP-9 as } \\
\text { biomarkers of endothelial dysfunction for } \\
\text { atherosclerotic plaque instability [22]. }\end{array}$ \\
\hline ANGPT L2 & $\begin{array}{l}\text { Endothelial cell-derived ANGPTL2 (a } \\
\text { proinflammatory mediator) }\end{array}$ & $\begin{array}{c}\text { An emerging candidate biomarker for CVD, } \\
\text { particularly atherosclerosis }[23,24] .\end{array}$ \\
\hline Endoglin & $\begin{array}{l}\text { CD105 or TGF- } \beta \text { receptor III (a homodimeric } \\
\text { transmembrane glycoprotein) }\end{array}$ & $\begin{array}{l}\text { Endoglin can be used as a biomarker of endothelial } \\
\text { dysfunction in CVD (e.g., hypercholesterolemia, } \\
\text { atherosclerosis, and hypertension) [25] and as a } \\
\text { possible marker of atorvastatin treatment benefit in } \\
\text { atherosclerosis [26]. }\end{array}$ \\
\hline Homocysteine & $\begin{array}{c}\text { A nonproteinogenic amino acid derived from } \\
\text { methionine }\end{array}$ & $\begin{array}{l}\text { Homocysteine is likely to act as an independent } \\
\text { predictor of adverse cardiovascular outcomes [27]; } \\
\text { baseline homocysteine concentration is likely to be a } \\
\text { predictor for the benefit of antihypertensive therapy } \\
\text { and a critical therapeutic target [28]. }\end{array}$ \\
\hline EMPs & $\begin{array}{l}\text { Annexin } \mathrm{V}^{+} \text {endothelial microparticles microparticles } \\
\text { are shed from apoptotic endothelial cells }\end{array}$ & $\begin{array}{l}\text { EMPs can be used to monitor inflammation and } \\
\text { thrombosis in the course of atherosclerosis [29]. }\end{array}$ \\
\hline
\end{tabular}

without a history of CVD. They showed that the age- and sex-adjusted incidence rate of CVD increased significantly and linearly with elevating serum ANGPTL2 concentrations. The elevating concentrations of serum ANGPTL2 were a significant risk factor for CVD after adjustment for conventional risk factors other than the metabolic syndrome components [24]. Hata et al. [24] suggest that elevated serum ANGPTL2 levels are a novel risk factor for the development of CVD in the general population. These studies indicate that ANGPTL2 potentially could be used as an emerging candidate biomarker for CVD, particularly atherosclerosis. However, data from a meta-analysis of ANGPTL2 of patients with atherosclerosis is still lacking in longitudinal study; therefore, further research is warranted to prove ANGPTL2 as a reliable biomarker.

Endoglin (CD105 or TGF- $\beta$ receptor III) is a homodimeric transmembrane glycoprotein that is mainly expressed in endothelial cells of blood vessels. A soluble form of endoglin is known as an extracellular domain of the fulllength membrane endoglin that is released into the blood circulation after proteolytic cleavage of the juxtamembrane region of membrane-bound endoglin under pathological conditions [25]. Increasing evidence is emerging that endoglin plays an important role in regulating cholesterolinduced endothelial dysfunction [25]. Recently Vicen et al. [7] showed that 7-ketocholesterol treated human aortic endothelial cells in atherosclerosis-prone apolipoprotein
E-deficient/LDL receptor-deficient (ApoE2/2/LDLR2/2) mice developed hypercholesterolemia in association with increased levels of P-selectin and endoglin as well as a disruption of NO metabolism. There appears to be an excellent correction between hypercholesterolemia-increased endoglin expression and endothelial dysfunction that is followed by developing atherosclerotic lesions by adhesion and transmigration of inflammatory cells via activated or dysfunctional endothelial cells [7]. In studies with apoE/LDL receptor (apoE/LDLR) double knockout mice, Strasky et al. [30] demonstrated that cholesterol-rich diet resulted in a significant increase of cholesterol and endoglin levels in serum, as well as increased plaque size of atherosclerosis in the aorta. Rathouska et al. [26] revealed that atorvastatin treatment is able to reduce the levels of cholesterol and plaque size as well as the levels of soluble form of endoglin. Rathouska et al. [25,26] proposed that increased the soluble form of endoglin can be used as a biomarker of endothelial dysfunction in CVD (e.g., hypercholesterolemia, atherosclerosis, and hypertension) and as a possible marker of atorvastatin treatment benefit in atherosclerosis.

It has demonstrated that homocysteine, a nonproteinogenic amino acid derived from methionine, is a strong predictor and independent risk factor for arterial ischemic events. In addition, it was reported the association of elevated homocysteine with metabolic syndrome and diabetes 
[31]. Wald et al. [27] showed that the serum concentration of the amino acid homocysteine was positively associated with the risk of ischemic heart disease, deep vein thrombosis and pulmonary embolism, and stroke. There is strong evidence from a meta-analysis that the association between homocysteine and cardiovascular disease is causal [27]. Thus, Wald et al. [27] suggest that lowering homocysteine concentrations by $3 \mu \mathrm{mol} / \mathrm{L}$ from current levels would reduce the risk of ischemic heart disease by $16 \%$, deep vein thrombosis by $25 \%$, and stroke by $24 \%$. These results indicate that the serum concentration of homocysteine is likely to act as an independent predictor of adverse cardiovascular outcomes [27]. A subsequent study showed that elevated homocysteine concentrations significantly decreased the antihypertensive effect of angiotensin-converting enzyme inhibitors (enalapril) in previously untreated hypertensive patients [28]. These results suggest that baseline homocysteine concentration is likely to be a predictor for the benefit of antihypertensive therapy and a critical therapeutic target [28]. There are several mechanisms by which homocysteine results in vascular damage, and one of them is induction of endothelial dysfunction [28]. Elevated homocysteine could promote angiotensin-converting enzyme (ACE) activity and act synergistically with angiotensin II to cause damage to the vasculature and lead to the deterioration of endothelial function [28]. Huang et al. [31] studied the role of homocysteinylation of ACE in endothelial dysfunction of coronary and mesenteric arteries obtained from male Wistar rats. They demonstrated that increased local/vascular production of homocysteine evoked endothelial dysfunction. The proposed mechanism by which elevated hyperhomocystinemia levels reduced enalapril efficacy is likely to be the direct effect of hyperhomocystinemia, which causes vascular dysfunction independent from renin-angiotensin system [31]. Taken tighter, these data provide the rationale of homocysteine as a candidate biomarker of endothelial dysfunction for cardiovascular diseases.

Circulating endothelial cells (Fig. 2) are detached as whole cells from mature endothelium under pathological conditions. The detached endothelial cells can be derived from reversibly activated endothelial cells (Fig. 3) or irreversibly endothelial apoptosis (Fig. 4) or necrosis [11,12]. Like circulating endothelial cells, circulating endothelial microparticles are shed from activated endothelial cells or apoptotic endothelial cells. Accordingly, circulating endothelial microparticles are emerging as a novel biomarker of endothelial activation and dysfunction for cardiovascular diseases. Elevated levels of E-selectin microparticles may represent early lesions of atherosclerosis, whereas elevated levels of annexin $\mathrm{V}$ microparticles reflect the presence of endothelial damage associated with inflammatory processes in accelerated atherosclerosis, thereby serving as a biomarker of endothelial dysfunction. On the other hand, circulating endothelial microparticles can be decreased with therapy [12]. There is growing evidence to support the dif- ferences of circulating endothelial microparticles between endothelial activation and dysfunction. Recently Rogula et al. [29] suggest that circulating endothelial microparticles can be used to monitor inflammation and thrombosis in the course of atherosclerosis, because endothelial extracellular vesicles in the circulation express various CDs (e.g., CD62E, CXD31, CD51, CD105, phosphatidylserine, and tissue factor) from activate or dysfunctional endothelium, and these CDs play roles in proinflammatory, proapoptotic, and procoagulant activities [29].

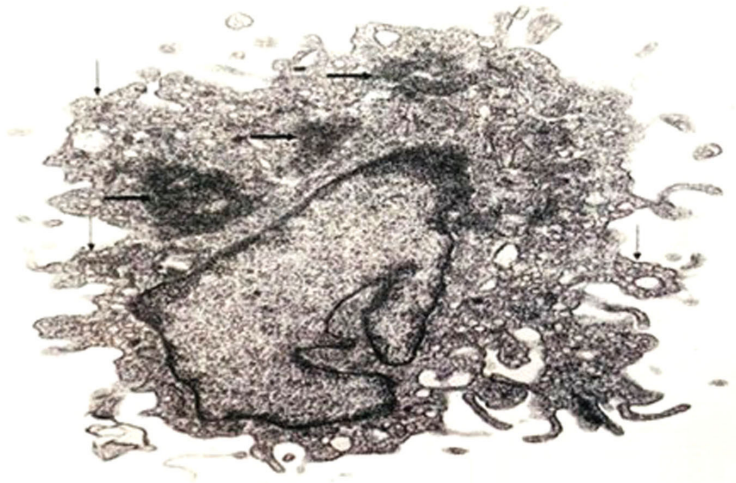

Fig. 2. Electron micrograph of circulating activated endothelial cell showing circulating activated-derived endothelial microparticles. A detached activated endothelial cell exhibited many long cytoplasmic processes, the flask-shaped invaginations on the surface of plasmalemmal membrane (vertical arrows), numerous vacuoles and vesicles in the cytoplasm, and enlarged Weibel-Palade bodies (horizontal arrows) that have a dense matrix housing tubular elements contained the glycoprotein von Willebrand factor. A cluster of discharging caveolae from the rest of the plasma membranes was found in the extracelluar space from activated endothelial cell that is consistent with EMPs. The characteristic of caveolae can be used to distinguish activated endothelial - derived microparticles from platelet- and leukocyte-derived microparticles. Original magnification, $\times 6300$. Adopted from Zhang J, Hanig JP, De Felice AF. Biomarkers of endothelial cell activation: candidate markers for drug-induced vasculitis in patients or drug-induced vascular injury in animals. Vascular Pharmacology. 2012; 56: 14-25.

Increased levels of annexin- $\mathrm{V}^{+}$microparticles were identified in patients with acute coronary syndrome, coronary artery disease, myocardial infarction, asymptomatic atherosclerosis, type II diabetes mellitus, and cardiopulmonary resuscitation [17,32]. Endothelial-derived microparticles were shown to be elevated after cardiopulmonary resuscitation in a study of Fink et al. [32]. They analyzed circulating endothelial-derived annexin $\mathrm{V}^{+}$microparticles in 36 patients after successful resuscitation, 20 


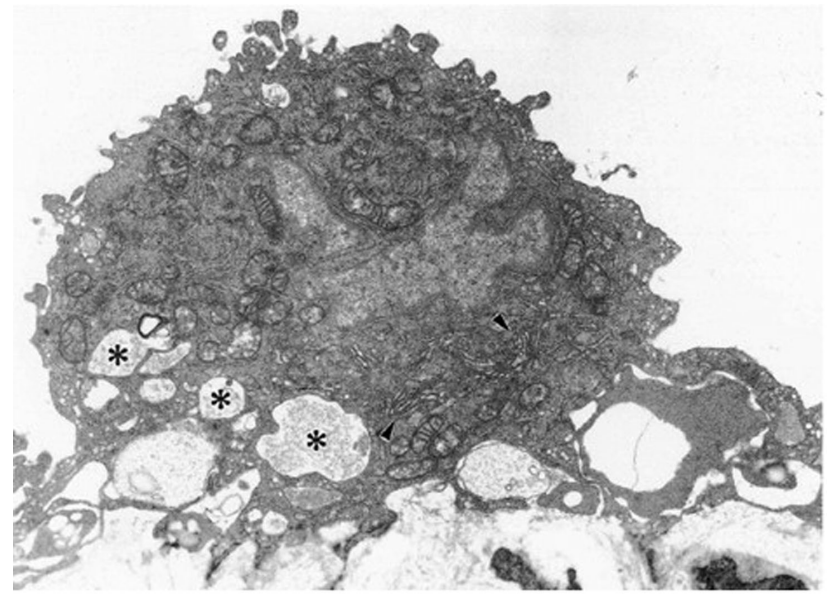

Fig. 3. Electron micrograph of activated endothelial cell showing cuboidal shape, well-develped Golgi complexes (arrowheads), vacuoles (asterisks), and numerous vesicles on the cell surface. Original magnification, $\times 8000$. Adopted from Zhang J, Herman EH, Knapton A, Chadwick DP, Whitehurst VE, Koerner JE, et al. SK\&F 95654-induced acute cardiovascular toxicity in Sprague-Dawley rats-Histopathologic, electron microscopic, immunohistochemical studies. Toxicogic Pathology. 2002; 30: 2840.

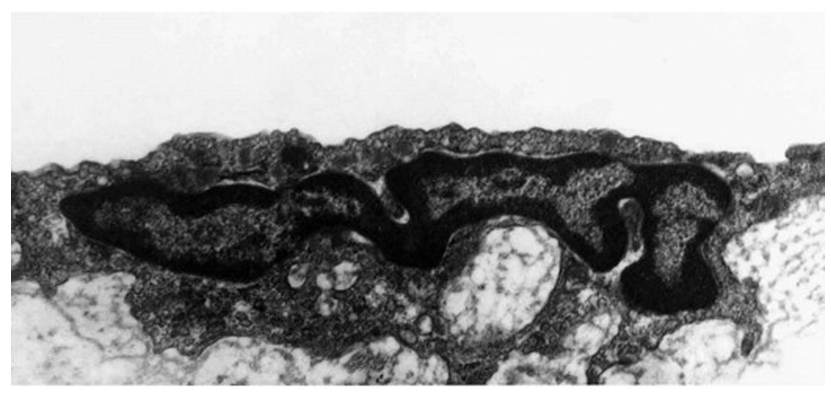

Fig. 4. Electron micrograph of apoptotic endothelial cell showing nuclear imagination and condensation, with numerous vesicles on the cell surface. Original magnification, $\times 4000$. Adopted from Zhang J, Herman EH, Knapton A, Chadwick DP, Whitehurst VE, Koerner JE, et al. SK\&F 95654-induced acute cardiovascular toxicity in Sprague-Dawley rats-Histopathologic, electron microscopic, immunohistochemical studies. Toxicogic Pathology. 2002; 30: 28-40.

control patients with stable cardiac disease and 15 healthy subjects. Substantially increased levels of endothelial annexin $\mathrm{V}^{+}$microparticles were found 24 hours after cardiopulmonary resuscitation in resuscitated patients. Furthermore, study of Fink et al. [32] also provides evidence for enhanced apoptosis of endothelial cells under the influence of microparticles of resuscitated patients ex vivo. Microparticles of cardiopulmonary resuscitation patients may contribute to endothelial apoptosis by conjugates pathway in which endothelial-derived microparticles inter- act with monocytes (endothelial microparticles-monocyte conjugates) or platelets (endothelial microparticles -platelet conjugates) to form circulating conjugates [32].

Endothelial dysfunction predicts morbidity and mortality in patients at cardiovascular risk. Werner et al. [33] assessed endothelial function and endothelial apoptosis in patients with coronary artery disease by quantification of circulating $\mathrm{CD} 31^{+}$/annexin $\mathrm{V}^{+}$apoptotic microparticles in peripheral blood. The study revealed the positive correlation of increased apoptotic microparticles counts with impairment of coronary endothelial function. Werner et al. [33] suggest that in patients with coronary artery disease, endothelial-dependent vasodilatation closely relies on the degree of endothelial apoptosis, which is readily measurable by circulating $\mathrm{CD} 31^{+} /$annexin $\mathrm{V}^{+}$apoptotic microparticles. Thus, increased apoptotic microparticle counts predict severe endothelial dysfunction independent of classical risk factors, such as hypertension, hypercholesterolemia, smoking, diabetes, age, or sex [33]. In the context of atherosclerosis, $\mathrm{CD} 31^{+}$/annexin $\mathrm{V}^{+}$microparticles are derived from platelets and the endothelium and they are increased in patients with cardiovascular risk factors. It was shown that endothelial dysfunction and consecutive atherosclerosis are based on a progressive loss of endothelial cells. Persistent endothelial damage and apoptosis due to classical cardiovascular risk factors result in the loss of the integrity of endothelium. As a result, an increased vascular permeability of the endothelium followed by inflammatory migration, and proliferation of vascular smooth muscle cells, an atherosclerotic lesion is developed. This provides the rationale for $\mathrm{CD} 31^{+}$/annexin $\mathrm{V}^{+}$microparticles involving in atherosclerosis. Sinning et al. [34] demonstrated that microparticle levels were significantly higher in patients with major adverse cardiovascular and cerebral event (MACCE) compared with patients without the event, and high levels of circulating $\mathrm{CD} 31^{+/}$annexin $\mathrm{V}^{+}$microparticles were associated with a higher risk for cardiovascular death. Therefore, Sinning et al. [34] suggest that the level of circulating CD31+/annexin $\mathrm{V}^{+}$microparticles is an independent predictor of cardiovascular events in patients with stable coronary artery disease patients and may be useful for risk stratification [33].

It is noteworthy that it is difficult to reconcile some controversial data about the circulating microparticle populations. In an observational study that consisted of 40 patients with an early stage of metabolic syndrome and 30 health controls [35]. Weight circumference, blood pressure, fasting blood glucose, and triglyceride were all statistically higher in these patients than in controls, however the percentage of endothelial microparticles in patients with metabolic syndrome was not increased compared with that in controls, but the percentage of platelet microparticles was significantly statistically different between the patients and control. The controversial results are needed to be elucidated. Whether this is due to the difference in clearance pe- 
riod between endothelial microparticles (short) and platelet microparticles (longer), or due to the difference in the original percentage of platelet derived microparticles (much abundant) and endothelial derived microparticles (less) remains unknown.

\section{Limitations of biomarkers of endothelial activation and dysfunction in clinical setting}

While biomarkers of endothelial activation and dysfunction play an important role in diagnosis of atherosclerosis, some limitations remain. For example, current technological limitations in purification of circulating microparticles remain, and standardized methods of microparticles detection is lacking [36,37]. Furthermore, like endothelial biomarkers of vascular injury in humans [38], endothelial biomarkers of cardiovascular disease in humans are usually not specific and are of limited sensitivity. Although they are useful to the clinician in identifying high-risk factors, diagnosing diseases, monitoring prognosis, and providing therapeutic intervention [38,39], some factors may limit these biomarkers for clinical use. Firstly, small example size in clinical studies may be a significant barrier to develop reliable biomarkers. Therefore, multicenter large clinical trials for evaluation are warranted. Secondly, as far as the sensitivity and specificity of a biomarker is concerned, it is critical to evaluate the information content of a biomarker over a range of values, often with the use of receiver operating characteristic (ROC) curves [39]. However, the methodology for this purpose was rarely used in these studies presented here. Thirdly, some data obtained from clinical studies are controversial and frequently under speculation. The mechanisms for dealing with contradiction are still lacking. In the context of circulating endothelial microparticles, using it as biomarker in everyday practice is also limited. This is likely the lack of standardized methods of microparticles [32,37,38]. Fourthly, endothelial-derived biomarkers are usually linked with other biomarkers from platelets, leukocytes, smooth muscle cells of blood vessels, and cardiac stem/progenitor cells. Without cooperation with other biomarkers, isolated use of endothelial biomarkers limits its application. Finally, less understanding of the differences in biomarkers between endothelial activation and dysfunction may also hinder to correctly interpret the development and outcome of diseases. Regardless of the limitations, some biomarkers may serve as research tools by providing insights into disease mechanisms [39] and they could be very promising candidate biomarkers of cardiovascular diseases.

\section{Discussion}

Biomarkers of endothelia activation and dysfunction include a heterogeneous group of candidate molecules. The data presented here briefly highlight some circulating soluble and cell component biomarkers of endothelial activation and dysfunction in CVD. Thus, the data presented here is fragmentary and incomplete. Regardless of this, this concise review could serve as a 'Continuing Medical Education' (CME) for clinicians. The primary concern of this review is, from pathological perspective, to distinguish biomarkers of endothelial activation from those of endothelial dysfunction. It is hoped that this distinction will bring clinicians accurately select the most relevant biomarkers to judge the development status of cardiovascular diseases. Furthermore, excellent conduct of clinical trials depends on a sound interpretation of the relationship of biomarkers of endothelial activation and dysfunction and clinical data, based on informed judgment and realistic understanding of development of cardiovascular diseases. Moreover, it is of most concern about the specificity and sensitivity of endothelial biomarkers. None of endothelial biomarkers has such property like cardiac troponin $\mathrm{T}$ or troponin I. Thus, it is wise and strategic that using combination of endothelial biomarkers will be better than single endothelial biomarker to make sense.

\section{Conclusions}

Understanding specific and sensitive biomarkers of endothelial activation and dysfunction may have broader clinical significance that could gain insight into pathogenesis for CVD, and the insight may lead to new approaches in treatment of CVD as well.

\section{Author contributions}

$\mathrm{JZ}$ designed and performed the research study, wrote the manuscript, contributed to editorial changes in the manuscript, and approved the final manuscript.

\section{Ethics approval and consent to participate}

Not applicable.

\section{Acknowledgment}

Not applicable.

\section{Funding}

This research received no external funding.

\section{Conflict of interest}

The author declares no conflict of interest. Jun Zhang is serving as one of the Editorial Board members of this journal. We declare that Jun Zhang had no involvement in the peer review of this article and has no access to information regarding its peer review. Full responsibility for the editorial process for this article was delegated to Carmela Rita Balistreri and Davide Bolignano.

\section{References}

[1] Gimbrone MA, García-Cardeña G. Endothelial Cell Dysfunction and the Pathobiology of Atherosclerosis. Circulation Research. 2016; 118: 620-636. 
[2] Baselet B, Sonveaux P, Baatout S, Aerts A. Pathological effects of ionizing radiation: endothelial activation and dysfunction. Cellular and Molecular Life Sciences. 2019; 76: 699-728.

[3] Deedwania, P. C. Mechanisms of endothelial dysfunction in the metabolic syndrome. Curr. Diab. Rep. 2003; 3, 289-292.

[4] Janus A, Szahidewicz-Krupska E, Mazur G, Doroszko A. Insulin Resistance and Endothelial Dysfunction Constitute a Common Therapeutic Target in Cardiometabolic Disorders. Mediators of Inflammation. 2016; 2016: 3634948.

[5] Hayden MR. Endothelial activation and dysfunction in metabolic syndrome, type 2 diabetes and coronavirus disease 2019. Journal of International Medical Research. 2020; 48: 030006052093974.

[6] Cotran RS, Pober JS. Endothelial activation. In Simionescu N, Simionescu M (eds.) Endothelial biology in health and diseases (pp. 335-347). Plenum Press: New York and London.1988.

[7] Vicen M, Vitverova B, Havelek R, Blazickova K, Machacek $\mathrm{M}$, Rathouska $\mathrm{J}$, et al. Regulation and role of endoglin in cholesterol-induced endothelial and vascular dysfunction in vivo and in vitro. FASEB Journal. 2019; 33: 6099-6114.

[8] Lundman P, Eriksson MJ, Silveira A, Hansson L, Pernow J, Ericsson $\mathrm{C}$, et al. Relation of hypertriglyceridemia to plasma concentrations of biochemical markers of inflammation and endothelial activation (C-reactive protein, interleukin-6, soluble adhesion molecules, von Willebrand factor, and endothelin-1). The American Journal of Cardiology. 2003; 91: 1128-1131.

[9] Drenjancevic I, Jukic I, Stupin A, Cosic A, Stupin M, SelthoferRelatie. The markers of endothelial activation. 2018.

[10] Rabelink TJ, de Boer HC, van Zonneveld AJ. Endothelial activation and circulating markers of endothelial activation in kidney disease. Nature Reviews Nephrology. 2010; 6: 404-414.

[11] Zhang J, Defelice AF, Hanig JP, Colatsky T. Biomarkers of endothelial cell activation serve as potential surrogate markers for drug induced vascular injury. Toxicologic Pathology. 2010; 38: 856-871.

[12] Zhang J, Hanig JP, De Felice AF. Biomarkers of endothelial cell activation: candidate markers for drug-induced vasculitis in patients or drug-induced vascular injury in animals. Vascular Pharmacology. 2012; 56: 14-25.

[13] Szmitko PE, Wang C, Weisel RD, de Almeida JR, Anderson TJ, Verma S. New markers of inflammation and endothelial cell activation: Part I. Circulation. 2003; 108: 1917-1923.

[14] Hwang SJ, Ballantyne CM, Sharrett AR, Smith LC, Davis CE, Gotto AM, et al. Circulating adhesion molecules VCAM-1, ICAM-1, and E-selectin in carotid atherosclerosis and incident coronary heart disease cases: the Atherosclerosis Risk in Communities (ARiC) study. Circulation. 1997; 96: 4219-4225.

[15] Tzoulaki I, Murray GD, Lee AJ, Rumley A, Lowe GDO, Fowkes FGR. C-reactive protein, interleukin-6, and soluble adhesion molecules as predictors of progressive peripheral atherosclerosis in the general population: Edinburgh Artery Study. Circulation. 2005; 112: 976-983.

[16] Cui Y, Zheng L, Jiang M, Jia R, Zhang X, Quan Q, et al. Circulating microparticles in patients with coronary heart disease and its correlation with interleukin-6 and C-reactive protein. Molecular Biology Reports. 2013; 40: 6437-6442.

[17] E. Berezin A. Impaired Phenotype of Circulating EndothelialDerived Microparticles: Novel Marker of Cardiovascular Risk. Journal of Cardiology and Therapy. 2015; 2: 365-370.

[18] Hu S, Zhang H, Zhang Q, Xiu R. Small-size circulating endothelial microparticles in coronary artery disease. PLoS ONE. 2014; 9: e104528.

[19] Landers-Ramos RQ, Addison OA, Beamer B, Katzel LI, Blumenthal JB, Robinson S, et al. Circulating microparticle concentrations across acute and chronic cardiovascular disease conditions. Physiological Reports. 2020; 15: e14534
[20] Tadzic R, Mihalj M, Vcev A, Ennen J, Tadzic A, Drenjancevic I. The effects of arterial blood pressure reduction on endocan and soluble endothelial cell adhesion molecules (CAMs) and CAMs ligands expression in hypertensive patients on $\mathrm{Ca}$ channel blocker therapy. Kidney \& Blood Pressure Research. 2013; 37: 103-115.

[21] Boumiza S, Chahed K, Tabka Z, Jacob M, Norel X, Ozen G. MMPs and TIMPs levels are correlated with anthropometric parameters, blood pressure, and endothelial function in obesity. Scientific Reports. 2021; 11: 20052.

[22] Olejarz W, Łacheta D, Ubiak-Tomaszewska G. Matrix metalloproteinases as biomarkers of atherosclerotic plaque instability. International Journal of Molecular Sciences. 2020; 21: 3946.

[23] Horio E, Kadomatsu T, Miyata K, Arai Y, Hosokawa K, Doi $\mathrm{Y}$, et al. Role of endothelial cell-derived angpt12 in vascular inflammation leading to endothelial dysfunction and atherosclerosis progression. Arteriosclerosis, Thrombosis, and Vascular Biology. 2014; 34: 790-800.

[24] Hata J, Mukai N, Nagata M, Ohara T, Yoshida D, Kishimoto H, et al. Serum Angiopoietin-Like Protein 2 is a Novel Risk Factor for Cardiovascular Disease in the Community. Arteriosclerosis, Thrombosis, and Vascular Biology. 2016; 36: 1686-1691.

[25] Rathouska J, Jezkova K, Nemeckova I, Nachtigal P. Soluble endoglin, hypercholesterolemia and endothelial dysfunction. Atherosclerosis. 2015; 243: 383-388.

[26] Rathouska J, Vecerova L, Strasky Z, Slanarova M, Brcakova E, Mullerova Z, et al. Endoglin as a possible marker of atorvastatin treatment benefit in atherosclerosis. Pharmacological Research. 2011; 64: 53-59.

[27] Wald DS, Law M, Morris JK. Homocysteine and cardiovascular disease: evidence on causality from a meta-analysis. British Medical Journal. 2002; 325: 1202.

[28] Qin X, Li Y, Sun N, Wang H, Zhang Y, Wang J, et al. Elevated Homocysteine Concentrations Decrease the Antihypertensive Effect of Angiotensin-Converting Enzyme Inhibitors in Hypertensive Patients. Arteriosclerosis, Thrombosis, and Vascular Biology. 2017; 37: 166-172.

[29] Rogula S, Gąsecka A, Filipiak KJ. Macroscopic role of microparticles in cardiovascular disease. Polski Merkuriusz Lekarski. 2020; 49: 255-259.

[30] Strasky Z, Vecerova L, Rathouska J, Slanarova M, Brcakova E, Kudlackova Z, et al. Cholesterol effects on endoglin and its downstream pathways in ApoE/LDLR double knockout mice. Circulation Journal. 2011; 75: 1747-1755.

[31] Huang A, Pinto JT, Froogh G, Kandhi S, Qin J, Wolin MS, et al. Role of homocysteinylation of ACE in endothelial dysfunction of arteries. American Journal of Physiology-Heart and Circulatory Physiology. 2015; 308: H92-H100.

[32] Fink K, Feldbrügge L, Schwarz M, Bourgeois N, Helbing T, Bode $\mathrm{C}$, et al. Circulating annexin $\mathrm{V}$ positive microparticles in patients after successful cardiopulmonary resuscitation. Critical Care. 2011; 15: R251.

[33] Werner N, Wassmann S, Ahlers P, Kosiol S, Nickenig G. Circulating CD31+/annexin $\mathrm{V}+$ apoptotic microparticles correlate with coronary endothelial function in patients with coronary artery disease. Arteriosclerosis, Thrombosis, and Vascular Biology. 2006; 26: 112-116.

[34] Sinning J-M, Losch J, Walenta K, Böhm M, Nickenig G, Werner N. Circulating CD311/Annexin V microparticles correlate with cardiovascular outcomes. European Heart Journal. 2011; 32: 2034-2041.

[35] Zahran AM, Sayed SK, Abd El Hafeez HA, Khalifa WA, Mohamed NA, Hetta HF. Circulating microparticle subpopulation in metabolic syndrome: relation to oxidative stress and coagulation markers. Diabetes, Metabolic Syndrome and Obesity. 2019; 12: 485-493. 
[36] Ardoin SP, Shanahan JC, Pisetsky DS. The role of microparticles in inflammation and thrombosis. Scandinavian Journal of Immunology. 2007; 66: 159-165.

[37] Barteneva NS, Fasler-Kan E, Bernimoulin M, Stern JNH, Ponomarev ED, Duckett L, et al. Circulating microparticles: square the circle. BMC Cell Biology. 2013; 14: 23.
[38] Kerns W, Schwartz L, Blanchard K, Burchiel S, Essayan D, Fung $\mathrm{E}$, et al. Drug-induced vascular injury-a quest for biomarkers. Toxicology and Applied Pharmacology. 2005; 203: 62-87.

[39] Vasan RS. Biomarkers of Cardiovascular Disease. Circulation. 2006; 113: 2335-2362. 